Pacific Journal of Mathematics

ON SOLVABILITY OF GENERALIZED ORTHOMODULAR Latices 


\title{
ON SOLVABILITY OF GENERALIZED ORTHOMODULAR LATTICES
}

\section{LADISLAV BERAN}

\begin{abstract}
The purpose of this paper is to establish a universal criterion for a generalized orthomodular lattice to belong to a primitive class of lattices.
\end{abstract}

The starting point for the investigation is the description of the reflection and the coreflection. These lattices can be determined by two lattice congruences defined by means of alleles on any lattice.

The main results permit to characterize a generalized orthomodular lattice solvable in a nontrivial primitive class $\mathscr{C}$ of lattices as a lattice belonging to $\mathscr{C}$. The characterization is further used to show that such a lattice belongs to $\mathscr{C}$ if and only if its commutator sublattice is a lattice of $\mathscr{C}$.

1. Preliminaries. Basic facts on orthomodular lattices used in this paper may be found in [4], and also most of the notation and terminology will be taken from that book. We assume familiarity with the results of Marsden [8] on solvability of generalized orthomodular lattices. The notation concerning the projectivity of quotients in a lattice is essentially the same as in [7].

Let $\Omega$ be a nonempty set of quotients of a lattice $\mathscr{L}$. A quotient $b / a$ is called an $\Omega$-allele of $\mathscr{L}$ if there exists $n \in \mathbf{N}$ and a sequence $b_{0} / a_{0}=b / a, b_{1} / a_{1}, \cdots, b_{n} / a_{n}$ of quotients of $\Omega$ such that $b_{i} / a_{i} \sim b_{i+1}^{\prime} / a_{i+1}^{\prime}$ for every $i=0,1, \cdots, n-1$ where $\left[a_{i+1}^{\prime}, b_{i+1}^{\prime}\right] \subset\left[a_{i+1}, b_{i+1}\right]$ and $b \leqq a_{n}$ or $a \geqq b_{n}$. The set $\mathrm{A}_{\Omega}(\mathscr{L})$ of all $\Omega$-alleles of $\mathscr{L}$ will be called the $\Omega$-allelomorph of the lattice $\mathscr{L}$.

For the special case $\Omega=\Omega_{0}=\{b / a \mid a \prec b$ ( $b$ covers $\left.a)\right\}$, the $\Omega_{0}$ alleles of a submodular lattice have been studied in the paper [1]. Other results concerned with $\Omega$-alleles can be found in [3].

In the present paper we shall investigate the $\Omega_{1}$-alleles where $\Omega_{1}$ is the set of all quotients of $\mathscr{L}$ and we shall omit reference to $\Omega_{1}$; e.g., we shall refer to an $\Omega_{1}$-allele as an allele; similarly, $\mathrm{A}(\mathscr{L})$ (or simply A) will be the set of all alleles. Hence, $b / a \in A(\mathscr{L})$ if and only if there exists $d / c$ such that $b / a$ is weakly projective into $d / c$, i.e. $b / a \approx_{w} d / c$, and $c \geqq b$ or $d \leqq a$.

2. Reflection and coreflection. For each lattice $\mathscr{L}$ there exists a smallest congruence $\Delta$ such that $\mathscr{L} / \Delta$ is distributive. Let $\Delta^{*}$ be the pseudocomplement of $\Delta$. We define the reflection of $\mathscr{L}$, denoted by $\operatorname{Ref} \mathscr{L}$, to be the lattice $\mathscr{L} / \Delta^{*}$; the lattice $\mathscr{L} / \Delta$ is denoted by 
Coref $\mathscr{L}$ and it is called the coreflection of $\mathscr{L}$. A well-known result asserts that a nontrivial primitive class of lattices contains the class of distributive lattices. Since $\mathscr{L}$ is a subdirect product of Ref $\mathscr{L}$ and Coref $\mathscr{L}$, it follows that a lattice belongs to a nontrivial class $\mathscr{C}$ of lattices if and only if its reflection belongs to $\mathscr{C}$.

The following lemma (which appears in $[9$, p. 95] as Lemma 2) is useful:

LEMMA 2.1. Let $\mathscr{L}$ be a lattice and $\theta$ a congruence of $\mathscr{L}$. If $[b] /[a] \approx_{w}[d] /[c]$ in $\mathscr{L} / \theta$, then there exists $\backslash a \in[a], \backslash b \in[b], \backslash c \in[c]$, $\backslash d \in[d]$ such that $\backslash b / a \approx_{w}{ }^{\prime} d / c$ in $\mathscr{L}$.

Proof. Suppose $[b] /[a]=\left[b_{0}\right] /\left[a_{0}\right]{ }_{w}\left[b_{1}\right] /\left[a_{1}\right] \sim_{w} \cdots \sim_{w}\left[b_{n}\right] /\left[a_{n}\right]=$ $[d] /[c]$ with $a_{0}=a \leqq b=b_{0}$ and $a_{n}=c \leqq d=b_{n}$. Let $\backslash d=b_{n}=d$ and $' c={ }^{\prime} a_{n}=c$, and suppose that ${ }^{\prime} a_{j},{ }^{\prime} b_{j}$ for $i<j<n$ have already been defined so that ${ }^{\prime} a_{j} \in\left[a_{j}\right], \quad b_{j} \in\left[b_{j}\right],{ }^{\prime} a_{j} \leqq \backslash b_{j}$, and $\backslash b_{j} / a_{j} \sim_{w}{ }^{\prime} b_{j+1} / a_{j+1}$. Then if $\left[b_{i}\right] /\left[a_{i}\right] /{ }_{w}\left[b_{i+1}\right] /\left[a_{i+1}\right]$, let ${ }^{\prime} a_{i}={ }^{\prime} a_{i+1} \wedge b_{i}, b_{i}=\backslash b_{i+1} \wedge b_{i}$, and if $\left[b_{i}\right] /\left[a_{i}\right] \searrow_{w}\left[b_{i+1}\right] /\left[a_{i+1}\right]$, let ${ }^{\prime} a_{i}={ }^{\prime} a_{i+1} \vee a_{i}, \quad b_{i}={ }^{\prime} b_{i+1} \vee a_{i}$. So we get ${ }^{\prime} a={ }^{\prime} a_{0} \in[a], \backslash b=b_{0} \in[b]$, and $\backslash b / a \approx_{w}{ }^{\prime} d / c$. Note that if $[a] \neq[b]$, we must have $a \neq b$.

COROLlary 2.2. Let $\mathscr{L}$ be a lattice and $\theta$ a congruence of $\mathscr{L}$. If $[b] /[a]$ is an allele of $\mathscr{L} / \theta$, then there exists $' a \in[a], \quad b \in[b]$ such that $\backslash b / a$ is an allele of $\mathscr{L}$.

Proof. Let $[b] /[a] \approx_{w}[d] /[c]$ with $a \leqq b \leqq c \leqq d$. Choose $\backslash a,{ }^{\prime} b,{ }^{\prime} c,{ }^{\prime} d$ as in Lemma 2.1. It suffices to prove that $b \leqq{ }^{\prime}$. But we may assume that $[b] /[a]=\left[b_{0}\right] /\left[a_{0}\right] /{ }_{w}\left[b_{1}\right] /\left[a_{1}\right]$, so $b=\backslash b_{0}=\backslash b_{1} \wedge b_{0}=\backslash b_{1} \wedge$ $b \leqq b \leqq c=' c$.

LEMma 2.3. (i) Let $\mathscr{L}$ be any lattice and let $a<b, r<s$ be elements of $\mathscr{L}$. Then if $b / a \approx_{w} s / r$ and $r=r_{0}<r_{1}<\cdots<r_{n}=s$, there exist $a_{j}, j=0,1, \cdots, n$ such that $a=a_{0} \leqq a_{1} \leqq \cdots \leqq a_{n}=b$ and $a_{j+1} / a_{j} \approx_{w} r_{j+1} / r_{j}$ for each $j$.

(ii) Let $\mathscr{L}$ be a lattice and let $\gamma$ be the binary relation defined on $L$ by

$$
\begin{aligned}
& a \equiv b(\gamma) \Leftrightarrow \exists n \in \mathrm{N} \exists a_{1}, a_{2}, \cdots, a_{n} \\
& a \wedge b=a_{0} \leqq a_{1} \leqq \cdots \leqq a_{n}=a \vee b,
\end{aligned}
$$

and

$$
a_{j+1} / a_{j} \in \mathrm{A}(\mathscr{L}) \text { for every } j=0,1, \cdots, n-1 .
$$

If $b / a \approx_{w} q / p$ and $p \equiv q(\gamma)$, then $a \equiv b(\gamma)$. 
Proof. (i) It is enough to consider the case $b / a \sim_{w} s / r$. Assume $b / a \searrow_{w} s / r$; then $a_{j}=a \vee r_{j}, j=0,1, \cdots, n$ are the required elements.

(ii) We will first treat the following two cases:

Case I. $\quad b / a \searrow_{w} q / p, q / p \approx_{w} s / r$ and $r \geqq q$. Let $r_{0}=r, r_{1}=r \vee$ $(b \wedge s)$ and $r_{2}=s$. Since $r_{1} / r \searrow b \wedge s / b \wedge r$ and $b / q \searrow a / a \wedge q$, we get that $r_{1} / r_{0} \approx_{w} a / a \wedge q$. We also have $r_{2} / r_{1} \approx_{w} b \vee s / b$. Since $b / a \approx_{w} s / r$, (i) implies that there exist $a_{0}=a \leqq a_{1} \leqq a_{2}=b$ such that $a_{1} / a_{0} \approx_{w} a / a \wedge q$ and $a_{2} / a_{1} \approx_{w} b \vee s / b$. Thus $a_{1} / a$ and $b / a_{1}$ belong to the allelomorph and so $a \equiv b(\gamma)$.

Case II. $b / a \nearrow_{w} q / p, q / p \approx_{w} s / r$ and $r \geqq q$. However, here we have $b / a \approx_{w} s / r$ and $r \geqq q \geqq p \vee b \geqq b$. This yields $b / a \in \mathrm{A}$ and, consequently, $a \equiv b(\gamma)$.

By the same argument as in the proof of (i) it is clear that $b / a \sim_{w} q / p$ and $p \equiv q(\gamma)$ implies $a \equiv b(\gamma)$. The general case now follows by induction.

Our next theorem provides much more information on $\gamma$.

THEOREM 2.4. Let $\mathscr{L}$ be a lattice. Then $\gamma$ is a congruence relation of $\mathscr{L}$.

Proof. If $x \leqq y, x \equiv y(\gamma)$ and $t \in L$, then $y \wedge t / x \wedge t \approx_{w} y / x$. By Lemma 2.3 (ii) it follows that $x \wedge t \equiv y \wedge t(\gamma)$. The conclusion is now immediate from [6, Lemma 8, p. 24].

The following theorem can be proved by similar arguments; we omit the proof.

THEOREM 2.5. Let $\mathscr{L}$ be a lattice and let $\beta$ be the binary relation defined on $L$ by

$$
a \equiv b(\beta) \Leftrightarrow\left\{\left(d / c \approx_{w} a \vee b / a \wedge b \& d / c \in \mathbf{A}(\mathscr{L})\right) \Rightarrow c=d\right\} .
$$

Then $\beta$ is a congruence of $\mathscr{L}$. Moreover, $\beta$ is the pseudocomplement of $\gamma$.

CoROllary 2.6. Let $\mathscr{L}$ be a weakly modular lattice. Then

$$
a \equiv b(\beta) \Leftrightarrow\{([m, n] \subset[a \wedge b, a \vee b] \& n / m \in \mathrm{A}(\mathscr{L})) \Rightarrow m=n\}
$$

Observe that the description of $\beta$ as given in Corollary 2.6 is a direct consequence of the proof of [7, Theorem III. 4. 10].

REMARK. If $\mathscr{L}$ is a relatively complemented lattice, then [5, 
p. 355] $y / x \approx_{w} b / a$ implies $y / x \approx b^{\prime} / a^{\prime}$ where $\left[a^{\prime}, b^{\prime}\right] \subset[a, b]$. We say that $b / a$ has a close allele $d / c$ if there exists a quotient $d / c$ such that $b / a \approx d / c \&(b \leqq c$ or $a \geqq d)$. Thus, in such a lattice $b / a \in A$ if and only if $b / a$ has a close allele and the congruences $\beta$ and $\gamma$ of $\mathscr{L}$ can be characterized in terms of close alleles.

Proposition 2.7. Let $\mathscr{L}$ be a lattice and let $\theta$ be a congruence relation of $\mathscr{L}$. Then the quotient lattice $\mathscr{L} \mid \theta$ is distributive if and only if $\theta \supset \gamma$.

Proof. If $\mathscr{L} / \theta$ is distributive and if $a \equiv b(\gamma)$, then there are elements $x_{i}$ such that

$$
a \wedge b=x_{0} \leqq x_{1} \leqq \cdots \leqq x_{s}=a \vee b
$$

and such that $x_{i+1} / x_{i} \in \mathrm{A}(\mathscr{L})$ for every $i=0,1, \cdots, s-1$. But then $\left[x_{i+1}\right] /\left[x_{i}\right] \in \mathrm{A}(\mathscr{L} / \theta)$ and by [7, Lemma III. 2.7] we have $\left[x_{i+1}\right]=\left[x_{i}\right]$. Thus $[a]=[b]$, i.e., $a \equiv b(\theta)$ and so $\gamma \subset \theta$.

To prove the converse, suppose $\mathscr{L} / \gamma$ is not distributive; then there exists an allele $[b] /[a]$ in $\mathscr{L} / \gamma$ such that $[a] \neq[b]$. By Corollary 2.2 there exists $\backslash a \in[a], \quad b \in[b]$ such that $b / / a$ is an allele in $\mathscr{L}$; hence $\backslash a \equiv b(\gamma)$ and so we get $[a]=[b]$, a contradiction.

The following is an application of Proposition 2.7.

CoROllaRy 2.8. If $\mathscr{L}$ is a lattice, then Coref $\mathscr{L}=\mathscr{L} / \gamma$ and $\operatorname{Ref} \mathscr{L}=\mathscr{L} \mid \beta$.

3. Solvability in classes of lattices. If $a, b$ are two elements of an ortholattice $\mathscr{L}=\left(L, \vee, \wedge,{ }^{\prime}, 0,1\right)$, then

$$
\operatorname{com}_{\mathscr{L}}(a, b)=(a \vee b) \wedge\left(a \vee b^{\prime}\right) \wedge\left(a^{\prime} \vee b\right) \wedge\left(a^{\prime} \vee b^{\prime}\right)
$$

is called the commutator of $a, b$ (cf. [8]). The $n$-th commutator sublattice $\mathscr{G}_{n}$ of a generalized orthomodular lattice $\mathscr{G}$ is defined by induction in the following way: $\mathscr{G}_{0}=\mathscr{G}$ and $\mathscr{G}_{n}(n \geqq 1)$ is by definition the $p$-ideal generated in $\mathscr{G}_{n-1}$ by all the commutators of the generalized orthomodular lattice $\mathscr{G}_{n-1}$. The lattice $\mathscr{G}$ is said to be solvable (in the sense of Marsden) if there exists $n \in \mathbf{N}$ such that $\mathscr{G}_{n}=\langle 0\rangle$. Recall that [8, Theorem 9, p. 361] $\mathscr{G}$ is solvable if and only if it is distributive.

It is easily verified that if we define $\mathscr{G}^{(0)}=\mathscr{G}$ and if $\mathscr{G}^{(n)}(n \geqq 1)$ equals to the ideal of the generalized orthomodular lattice $\mathscr{G}$ generated by all the commutators of $\mathscr{G}^{(n-1)}$, then $\mathscr{G}_{n}=\mathscr{G}^{(n)}$ for every $n=$ $0,1, \cdots$. A generalized orthomodular lattice $\mathscr{G}$ is said to be solvable in a class $\mathscr{C}$ of lattices if and only if there exists $n \in \mathbb{N}$ such that 
$\mathscr{G}^{(n)}$ belongs to $\mathscr{C}$.

Since it is known [8, Theorem 9, p. 361] that $\mathscr{G}_{1}=\mathscr{G}_{2}=\ldots$, it is clear that in the definitions above we can suppose $n=1$. We remark that it is a simple matter to verify that a lattice $\mathscr{G}$ is solvable in the class of distributive lattices if and only if it is solvable in the sense of Marsden.

The solvability of generalized orthomodular lattices in the class of modular lattices has been investigated in [2].

Proposition 3.1. Let $\mathscr{G}$ be a generalized orthomodular lattice. Then an element a of $\mathscr{G}$ belongs to $\mathscr{G}^{\prime}$ if and only if $a \equiv 0(\gamma)$.

Proof. By [8, Theorems 6 and 7, p. 360], the commutator sublattice $\mathscr{G}^{\prime}$ is the kernel of the congruence $\Delta$. The conclusion $\gamma=\Delta$ is now immediate from Proposition 2.7.

THEOREM 3.2. The commutator sublattice $\mathscr{G}^{\prime}$ of a generalized orthomodular lattice $\mathscr{G}$ is a dually distributive ideal.

Proof. To prove the assertion, it is sufficient to show that if $I_{1}, I_{2}$ are ideals of $\mathscr{G}$, then necessarily $G^{\prime} \cap\left(I_{1} \vee I_{2}\right) \subset\left(G^{\prime} \cap I_{1}\right) \vee$ $\left(G^{\prime} \cap I_{2}\right)$. Suppose $m \in G^{\prime}$ and $m \leqq i_{1} \vee i_{2}, i_{s} \in I_{s}(s=0,1)$. Let $x^{\prime}$ denote the orthocomplement of an element $x \in\left[0, i_{1} \vee i_{2}\right]$ in the orthomodular lattice determined by the interval $\left[0, i_{1} \vee i_{2}\right]$.

Set

$$
\begin{aligned}
& k_{1}=i_{1} \wedge\left(i_{1}^{\prime} \vee m \vee i_{2}\right) \wedge\left(i_{1}^{\prime} \vee m \vee i_{2}^{\prime}\right), \\
& k_{2}=i_{2} \wedge\left(i_{2}^{\prime} \vee m \vee i_{1}\right) \wedge\left(i_{2}^{\prime} \vee m \vee i_{1}^{\prime}\right)
\end{aligned}
$$

so that $k_{s}=i_{s} \wedge c \in I_{s}$ where

$$
c=\left(i_{1}^{\prime} \vee m \vee i_{2}\right) \wedge\left(i_{1}^{\prime} \vee m \vee i_{2}^{\prime}\right) \wedge\left(i_{2}^{\prime} \vee m \vee i_{1}\right) .
$$

Let $w=i_{1} \wedge\left(i_{1}^{\prime} \vee m\right)$ and $v=i_{1} \wedge m$. Since $v \leqq m \in G^{\prime}, v \in G^{\prime}$. Now we have $w \wedge v^{\prime}=i_{1} \wedge \operatorname{com}\left(i_{1}, m\right) \in G^{\prime}$ and so $w=v \vee\left(w \wedge v^{\prime}\right) \in G^{\prime}$.

Let $w^{+}=w^{\prime} \wedge k_{1}$. Since $i_{1}$ commutes with $i_{1}^{\prime}$ and $i_{1} \wedge m^{\prime}$, it follows that $\left[i_{1}^{\prime} \vee\left(i_{1} \wedge m^{\prime}\right)\right] \wedge i_{1}=i_{1} \wedge m^{\prime}$. Thus $w^{+} \leqq \operatorname{com}\left(i_{1}^{\prime} \vee m, i_{2}\right)$ and this yields $w^{+} \in G^{\prime}$. Therefore $k_{1}=w \vee w^{+} \in G^{\prime} \cap I_{1}$ and, by symmetry, $k_{2} \in G^{\prime} \cap I_{2}$.

It is clear that $m \leqq\left(i_{1} \vee i_{2}\right) \wedge c$ and that every $i_{s}$ commutes with each element $(\cdots)$ of the definition of the element $c$. Therefore, $i_{1}$ and $i_{2}$ commute with $c$ and this gives

$$
m \leqq\left(i_{1} \vee i_{2}\right) \wedge c=\left(i_{1} \wedge c\right) \vee\left(i_{2} \wedge c\right)=k_{1} \vee k_{2} .
$$

This shows that $m \in\left(G^{\prime} \cup I_{1}\right) \vee\left(G^{\prime} \cap I_{2}\right)$, completing the proof. 
COROLlaRY 3.3. The ideal $\mathscr{G}^{\prime}$ of a generalized orthomodular lattice $\mathscr{G}$ is neutral.

Proof. By Proposition 3.1 and Theorem III. 3.10 of [7], the ideal $\mathscr{G}^{\prime}$ is standard. By Theorem 3.2 and Theorem III. 2.5 of [7] we see that $\mathscr{G}^{\prime}$ is neutral.

For an element $g \in G$ let $[g]$ denote the corresponding element of the quotient lattice $\mathscr{G} / \beta$ where $\mathscr{G}$ is a generalized orthomodular lattice and let $K([g])=(g] \cap G^{\prime}$. If $g_{1} \in[g]$ and $k \in K([g])$, then $k \equiv$ $k \wedge g_{1} \wedge g(\gamma)$. Since $k / k \wedge g_{1} \wedge g \nearrow_{w} g / g_{1} \wedge g$ and $g \equiv g_{1} \wedge g(\beta)$, we have $k \equiv k \wedge g_{1} \wedge g(\beta)$. By Theorem 2.5 this implies $k \leqq g_{1} \wedge g \leqq g_{1}$. The set $K([g])$ is therefore well-defined.

LEMMA 3.4. Under the convention made above we have

(i) $[g]=\sup _{\mathscr{G | \beta}}\{[k] \mid k \in K([g])\}$;

(ii) $K\left(\left[g_{1} \wedge g_{2}\right]\right)=K\left(\left[g_{1}\right]\right) \wedge K\left(\left[g_{2}\right]\right)$

$K\left(\left[g_{1} \vee g_{2}\right]\right)=K\left(\left[g_{1}\right]\right) \vee K\left(\left[g_{2}\right]\right)$

where the symbols $\vee, \wedge$ of the right-hand side denote the join and the meet in the ideal lattice $\mathscr{T}$ of the lattice $\mathscr{G}^{\prime}$;

(iii) the mapping $f:[g] \mapsto K([g])$ is an isomorphism of the lattice $\mathscr{G} \mid \beta$ onto a sublattice of the lattice $\mathscr{T}$.

Proof. (i) If $[h]$ is such that $[h]<[g]$ with $h<g$, then, by Corollary 2.6, there are $v, w$ satisfying $h \leqq v<w \leqq g$ and $w / v \in \mathrm{A}$. Let $z$ denote a complement of $v$ in $[0, w]$. Then $v \equiv w(\gamma)$ implies $z \in G^{\prime}$. If $[z] \leqq[h]$, then $[v]=[w]$ and so $v \equiv w(\beta \cap \gamma)$. Therefore, by Theorem 2.5, $v=w$, a contradiction. Since $z \in K([g])$, we see that $[h]$ is not an upper bound for the set $\{[k] \mid k \in K([g])\}$. Conclusion (i) results.

(ii) This is immediate by Theorem 3.2.

Assertion (iii) now follows directly from (i) and (ii) above.

THEOREM 3.5. Let $\mathscr{C}$ be a primitive class of lattices which contains a lattice with more than one element. Then a generalized orthomodular lattice is a lattice of $\mathscr{C}$ if and only if its commutator sublattice belongs to $\mathscr{C}$.

COROLLARY 3.6. A generalized orthomodular lattice is solvable in a primitive class $\mathscr{C}$ which contains a lattice with more than one element if and only if it belongs to the class $\mathscr{C}$.

Proof. If $\mathscr{G}$ belongs to $\mathscr{C}$, then its sublattice $\mathscr{G}^{\prime}$ belongs also to $\mathscr{C}$. 
Conversely, suppose $\mathscr{G}^{\prime} \in \mathscr{C}$. By [6, Lemma 8, p. 34], the ideal lattice $\mathscr{T}$ of $\mathscr{G}^{\prime}$ also belongs to the class $\mathscr{C}$. Since $\mathscr{C}$ is primitive, every isomorphic image of a sublattice of $\mathscr{T}$ belongs to $\mathscr{C}$. It, therefore, follows from Lemma 3.4 (iii) that ref $\mathscr{G} \in \mathscr{C}$. It is now immediate that $\mathscr{G}$ belongs to $\mathscr{C}$.

The author wishes to thank the referee for suggestions on the formulation of the theorems in $\S 2$ and particularly for his pointing out the consequence stated in Corollary 3.3 as well as for his many other valuable comments.

\section{REFERENCES}

1. L. Beran, Treillis sous-modulaires, Séminaire Dubreil-Pisot: Algèbre et théorie des nombres, Fac. Sci. Paris, 21e année, (1967/68), n 13, p. 13.01-13.17.

2. - Modularity in generalized orthomodular lattices, Comment. Math. Univ. Carolinae 15, No. 1 (1974), 189-193.

3. - Reflection and coreflection in generalized orthomodular lattices (to appear).

4. G. Birkhoff, Lattice Theory, Amer. Math. Soc. Colloq. Publ. Vol. XXV, Providence, R. I. (1967).

5. R.P. Dilworth, The structure of relatively complemented lattices, Ann. of Math., 51 (1950), 348-359.

6. G. Grätzer, Lattice Theory (First Concepts and Distributive Lattices), W.H. Freeman, San Francisco 1971.

7. — Lattice Theory (Second Part) (to appear).

8. E. L. Marsden, Jr., The commutator and solvability in a generalized orthomodular lattice, Pacific J. Math., 33 (1970), 357-361.

9. R. Wille, Primitive subsets of lattices, Algebra Universalis, 2 (1972), 95-98.

Received February 28, 1974 and in revised form November 11, 1974.

Charles University, Prague

SokolovsKá 83 Czechoslovakia 



\section{PACIFIC JOURNAL OF MATHEMATICS}

\section{EDITORS}

RICHARD ARENS (Managing Editor)

University of California

Los Angeles, California 90024

\section{J. DugundJI}

Department of Mathematics University of Southern California Los Angeles, California 90007

D. Gilbarg and J. Milgram

Stanford University

Stanford, California 94305
University of Washington Seattle, Washington 98105

\section{ASSOCIATE EDITORS}
E. F. BECKENBACH
B. H. NeumanN
F. WolF
K. YoShIDA

\section{SUPPORTING INSTITUTIONS}

\author{
UNIVERSITY OF SOUTHERN CALIFORNIA \\ STANFORD UNIVERSITY \\ UNIVERSITY OF TOKYO \\ UNIVERSITY OF UTAH \\ WASHINGTON STATE UNIVERSITY \\ UNIVERSITY OF WASHINGTON \\ $\stackrel{*}{*} \stackrel{*}{*} \stackrel{*}{ }{ }^{*}$ AMERICAN MATHEMATICAL SOCIETY
}

The Supporting Institutions listed above contribute to the cost of publication of this Journal, but they are not owners or publishers and have no responsibility for its content or policies.

Mathematical papers intended for publication in the Pacific Journal of Mathematics should be in typed form or offset-reproduced, (not dittoed), double spaced with large margins. Underline Greek letters in red, German in green, and script in blue. The first paragraph or two must be capable of being used separately as a synopsis of the entire paper. Items of the bibliography should not be cited there unless absolutely necessary, in which case they must be identified by author and Journal, rather than by item number. Manuscripts, in triplicate, may be sent to any one of the editors. Please classify according to the scheme of Math. Reviews, Index to Vol. 39. All other communications should be addressed to the managing editor, or Elaine Barth, University of California, Los Angeles, California, 90024.

The Pacific Journal of Mathematics expects the author's institution to pay page charges, and reserves the right to delay publication for nonpayment of charges in case of financial emergency.

100 reprints are provided free for each article, only if page charges have been substantially paid. Additional copies may be obtained at cost in multiples of 50 .

The Pacific Journal of Mathematics is issued monthly as of January 1966. Regular subscription rate: $\$ 72.00$ a year (6 Vols., 12 issues). Special rate: $\$ 36.00$ a year to individual members of supporting institutions.

Subscriptions, orders for back numbers, and changes of address should be sent to Pacific Journal of Mathematics, 103 Highland Boulevard, Berkeley, California, 94708.

\section{PUBLISHED BY PACIFIC JOURNAL OF MATHEMATICS, A NON-PROFIT CORPORATION}

Printed at Kokusai Bunken Insatsusha (International Academic Printing Co., Ltd.), 270, 3-chome Totsuka-cho, Shinjuku-ku, Tokyo 160, Japan.

\section{Copyright (C) 1975 by Pacific Journal of Mathematics} Manufactured and first issued in Japan 


\section{Pacific Journal of Mathematics}

\section{Vol. 57, No. $2 \quad$ February, 1975}

Norman Larrabee Alling, On Cauchy's theorem for real algebraic curves with boundary .......

Daniel D. Anderson, A remark on the lattice of ideals of a Prüfer domain ..................

Dennis Neal Barr and Peter D. Miletta, A necessary and sufficient condition for uniqueness of

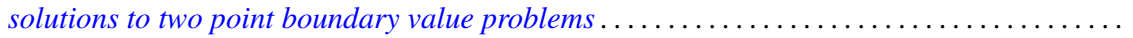

Ladislav Beran, On solvability of generalized orthomodular lattices . . . . . . . . . . ........

L. Carlitz, A three-term relation for some sums related to Dedekind sums . . . . . . . . . .....

Arthur Herbert Copeland, Jr. and Albert Oscar Shar, Images and pre-images of localization

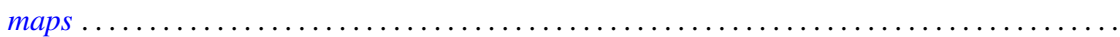

G. G. Dandapat, John L. Hunsucker and Carl Pomerance, Some new results on odd perfect

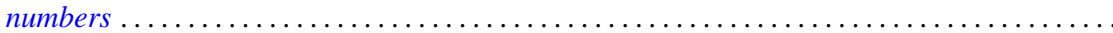

M. Edelstein and L. Keener, Characterizations of infinite-dimensional and nonreflexive

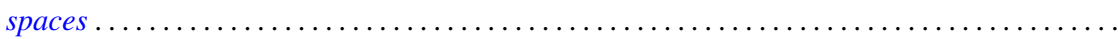

Francis James Flanigan, On Levi factors of derivation algebras and the radical embedding

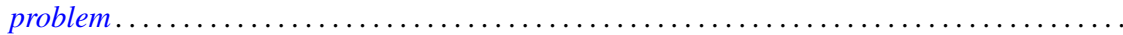

Harvey Friedman, Provable equality in primitive recursive arithmetic with and without

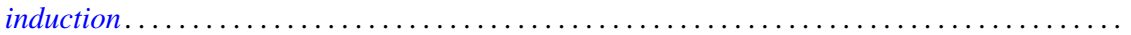

Joseph Braucher Fugate and Lee K. Mohler, The fixed point property for tree-like continua with

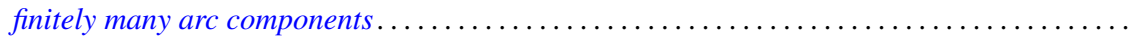

John Norman Ginsburg and Victor Harold Saks, Some applications of ultrafilters in

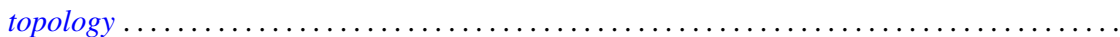

Arjun K. Gupta, Generalisation of a "square" functional equation .....................

Thomas Lee Hayden and Frank Jones Massey, Nonlinear holomorphic semigroups ..........

V. Kannan and Thekkedath Thrivikraman, Lattices of Hausdorff compactifications of a locally

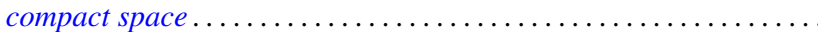

J. E. Kerlin and Wilfred Dennis Pepe, Norm decreasing homomorphisms between group

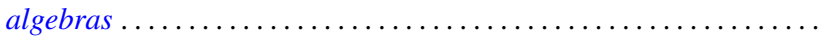

Young K. Kwon, Behavior of $\Phi$-bounded harmonic functions at the Wiener boundary ...

Richard Arthur Levaro, Projective quasi-coherent sheaves of modules .

Chung Lin, Rearranging Fourier transforms on groups...........................

David Lowell Lovelady, An asymptotic analysis of an odd order linear differential equation . . 4475

Jerry Malzan, On groups with a single involution .......................... 481

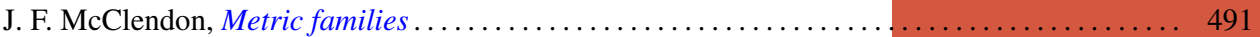

Carl Pomerance, On multiply perfect numbers with a special property .

Mohan S. Putcha and Adil Mohamed Yaqub, Polynomial constraints for finiteness of semisimple rings. .

Calvin R. Putnam, Hyponormal contractions and strong power convergence . . . . . . . . . 531

Douglas Conner Ravenel, Multiplicative operations in $\mathrm{BP} * \mathrm{BP} \ldots \ldots \ldots \ldots \ldots \ldots \ldots \ldots \ldots .539$

Judith Roitman, Attaining the spread at cardinals which are not strong limits . . . . . . . . . 545

Kazuyuki Saitô, Groups of *-automorphisms and invariant maps of von Neumann algebras . . . 553

Brian Kirkwood Schmidt, Homotopy invariance of contravariant functors acting on smooth

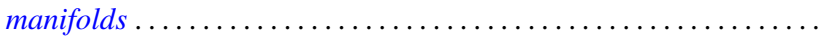

Kenneth Barry Stolarsky, The sum of the distances to $N$ points on a sphere.

Mark Lawrence Teply, Semiprime rings with the singular splitting property.

J. Pelham Thomas, Maximal connected Hausdorff spaces..............

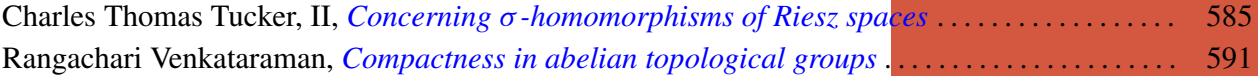

William Charles Waterhouse, Basically bounded functors and flat sheaves . . . . . . . . . . . 597

David Westreich, Bifurcation of operator equations with unbounded linearized part ......... 611

William Robin Zame, Extendibility, boundedness and sequential convergence in spaces of 